\title{
Medical Nutrition Support for Renal Patients and Young Athletes
}

\author{
KIYOTAKA YABUKI* \\ *Director of Yabuki Hospital, Yamagata, Japan
}

\begin{abstract}
An appropriate dietary intake is critical to sustaining health and performance in chronic disease patients and highly active individuals such as athletes. Research has shown that chronic renal failure patients benefit greatly by complementing traditional medical treatments with medical nutrition therapy (MNT). The role of MNT is to reduce the risk of complications in pre-existing conditions as well as ameliorating any existing conditions. For highly active athletes it serves as preventative care.

Yabuki hospital has created an original MNT system for renal failure patients, high school athletes and a local restaurant. The MNT program involves a nutritional assessment of patient's or athlete's dietary patterns coupled with results of laboratory testing. Other aspects of the program include conducting daily or monthly one-on-one consultations with patients, serving low-salt, low-fat meals, and hosting cooking classes for renal patients and their families in the in-hospital restaurant and kitchen. For athletes, those with problematic assessments are counseled by a dietician with their parents present.

One result of the program is that renal patients who eat MNT meals provided by the in-hospital restaurant are less likely to gain weight. As well, both athletes and renal patients are more likely to comply with their recommended diets on their own.
\end{abstract}

Key words: medical nutrition therapy (MNT), nutrition support, chronic renal failure (CRF), dialysis, high school athletes

\section{Introduction}

Many medical conditions either develop or worsen due to improper or unhealthy diets. As such, an appropriate dietary intake is important not only for patients with chronic diseases, but also for highly active individuals such as athletes. Medical nutrition therapy (MNT) seeks to use individually tailored diets to alleviate or treat medical conditions and their accompanying symptoms under the supervision of a registered dietitian (RD) or nutritionist. While medical institutions readily acknowledge the importance of nutritional support especially for patients with chronic diseases, few have dedicated facilities to provide this support and can provide patients only with advice or written materials.

Yabuki Hospital (Figure-1), located in Yamagata Prefecture, specializes in renal diseases. The hospital houses 40 beds and 84 dialysis consoles and primarily treat patients with chronic renal failure $(\mathrm{CRF})$ on an inpatient and outpatient basis. CRF patients require nutrition therapy and lifestyle changes that are outside the scope of a doctor's care

\author{
Kiyotaka Yabuki \\ Yabuki Hospital \\ 4-5-5 Shimakita, Yamagata-shi, Yamagata 990-0885, Japan \\ TEL: +81-23-682-8566 (ext. 3301) E-mail: k.yabuki@jeans.ocn.ne.jp \\ 339th Triannual Meeting of the Juntendo Medical Society: Medical Research Update〔Held on May 21, 2016〕 \\ 〔Received July 15, 2016]
}

Copyright (C) 2017 The Juntendo Medical Society. This is an open access article distributed under the terms of Creative Commons Attribution License (CC BY), which permits unrestricted use, distribution, and reproduction in any medium, provided the original source is properly credited. doi: $10.14789 /$ jmj. 63.22 


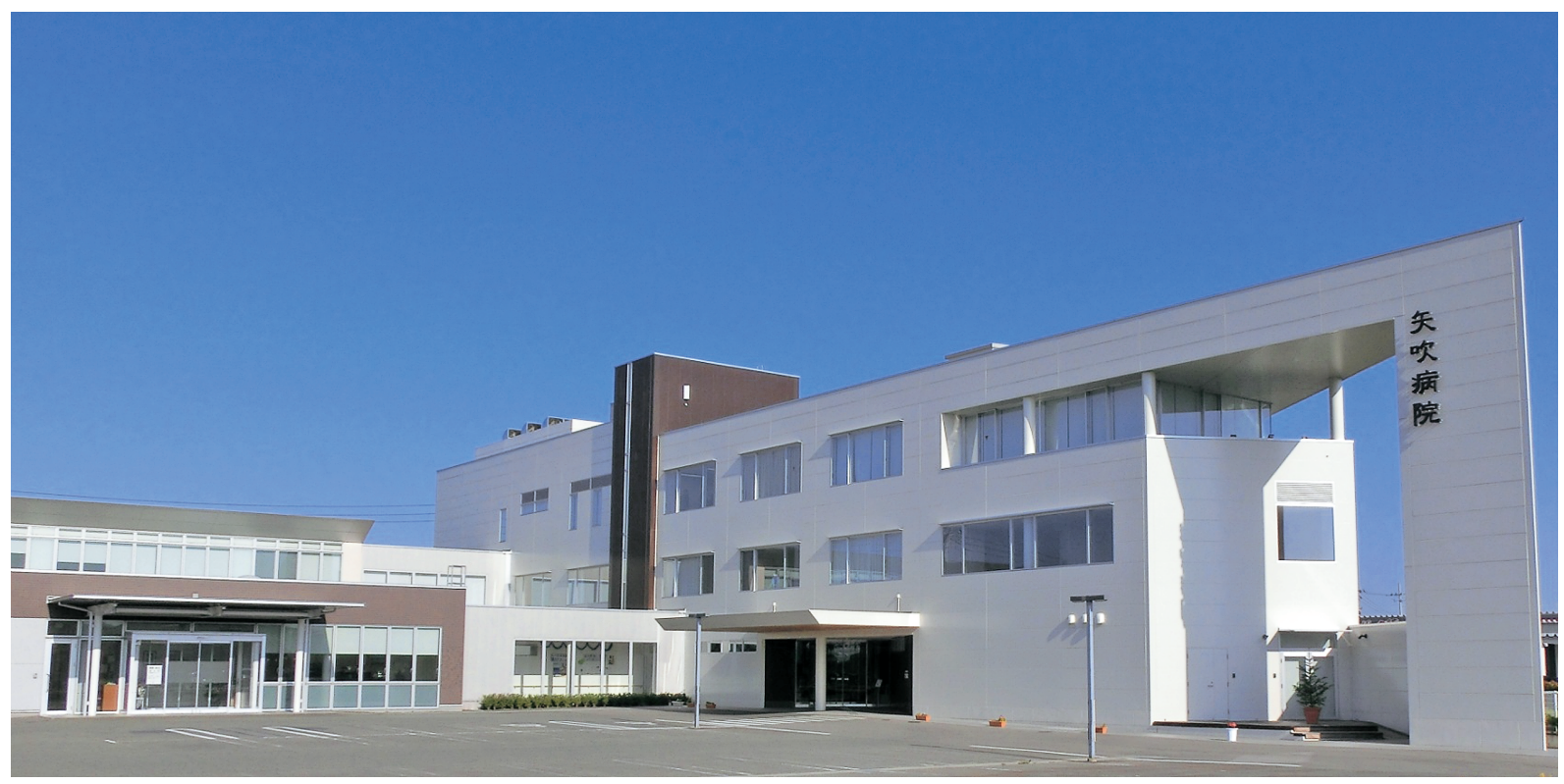

Figure-1 Yabuki Hospital

and are challenging for patients to implement on their own. In particular, many patients find it difficult to comply with dietary recommendations at home even though complying would help to manage their condition and improve their quality of life. MNT helps to overcome these challenges since registered dieticians and nutritionists supervise the program and are trained to assess individual nutritional needs, and create diets for specific medical conditions. They work with CRF patients individually and this type of support increases patients' compliance with dietary recommendations. At Yabuki Hospital we currently have six registered dieticians in the MNT program.

MNT can also be effective as a preventative measure. In a randomized study, the Diabetes Prevention Program reported a 58\% reduction over three years in the incidence of diabetes among 1,079 participants aged 25 to 84 years who underwent lifestyle changes that included $\mathrm{MNT}^{1}{ }^{1}$. As part of a preventive outreach, we have experimented with nutritional support programs outside the hospital for high school athletes and for a local French restaurant.

This case study looks at the various components of the current MNT program at Yabuki hospital. The program consists mainly of an in-hospital program for CRF patients and two small initiatives that seek to assist high school athletes and provide healthier menu options for a local French restaurant.

\section{Medical nutrition therapy for patients with chronic renal failure}

\section{Outpatients}

Approximately 60 non-dialysis dependent (NDD) CRF patients visit our hospital once a month on an outpatient basis. NDD CRF patients are persons with established chronic kidney disease who do not yet require the life supporting treatments for renal failure such as dialysis or renal transplant. Support for these patients is aimed at delaying disease progression protecting their kidneys. On the day of their monthly visit NDD CRF patients undergo blood and urine test, then, their required daily sodium and protein intake are estimated. In the patients with higher than normal weight gain a body constituent analysis is performed. Patients also have a one-on-one consul with a dietician to check their compliance to the recommended low-sodium and low-protein diet and to receive adjustments to their diet if the test results prove it necessary.

End-stage renal disease, is the final stage of chronic kidney disease where the kidneys stop working and a patient cannot survive without dialysis or a kidney transplant. Patients at this stage typically receive either hemodialysis (HD) administered using an artificial kidney on an out-patient basis at the hospital or peritoneal dialysis selfadministered at home. At Yabuki Hospital, HD is 
usually administered for 4 hours a day, 3 times a week. Dietitians visit the dialysis room daily at lunch time and give HD outpatients nutrition guidance following the guidelines of The Japanese Society for Dialysis Therapy ${ }^{2)}$. Dietary recommendations for patients are individualized based on changes in their body weight and blood pressure as well as results of monthly blood tests. For these end-stage renal patients, particularly the elderly, adverse health changes are usually triggered by weight gain, excessive intake of potassium or phosphorus, and eating disorders. Every six months, patients complete a general nutrition survey based on the Malnutrition Inflammation Score ${ }^{3)}$ 4) and undergo a monthly blood test. Information derived from patients' blood test, questionnaire, and lunch time counsel are pooled and discussed among the physicians, dietitians, nurses and clinical engineers so they can get an accurate picture of each patient's clinical state, and living conditions. The MNT team assesses the dietary needs of patients, identify negative changes in relevant blood markers and decide if changes are to be made to the patient's recommended dietary intake. Based on this the best treatment strategy is determined for each patient. We also recommend that patients have dietitian-supervised meals at our in-hospital restaurant, to make it easy to comply with their diets.

Peritoneal dialysis (PD) is conducted by injecting dialysate fluids into the patient's intraperitoneal space, retaining it for a certain amount of time, and flushing out the fluids and the toxins. PD patients receive dialysis at home daily and make monthly visits to Yabuki hospital for general checkups, and to receive nutritional support based on The Japanese Society for Dialysis Therapy guidelines ${ }^{2)}$. As many patients are over 70 years old, a decline in physical activity and forgetting to have meals due to dementia, are common occurrences. In addition to one-on-one consultations with a dietitian, patients are encouraged to participate in hospital events such as luncheons and patient group meetings.

\section{Inpatients}

Patients committed to the hospital tend to have $\mathrm{CRF}$, and the clinical status varies among patients. MNT for renal failure patients considers the patient's overall status but extends beyond that to consider their ability to swallow, physical activity level, existence of dementia and clinical response to dialysis and even their food preference. Daily MNT for admitted patients starts with the morning hand over when the clinician who worked the night shift is relieved. A ward dietitian is usually present for the shift change and observes as the clinician checks for changes in each patient's vitals. Again during lunchtime, the dietitian visits patients' to check on their food intake. Physicians and dietitians consistently exchange information about patients' clinical states and their dietary intake. In addition, a weekly meeting is held where physicians, dietitians, nurses, pharmacists, physiotherapists and certified social workers can bring each other up-to-date on patient related issues, concerns, and give suggestions. The same team members also participate in a monthly nutrition support meeting to discuss initiatives to improve the hospital's MNT initiative. In order to provide tasty hospital meals, not only dietitians but also physicians, nurses and other members from the MNT committee participate in tasting meals prepared at the hospital and give their feedback on the dishes.

\section{Facilities for medical nutrition therapy at Yabuki Hospital}

\section{In-hospital restaurant}

Patients with lifestyle-related diseases such as hypertension or hyperlipidemia are usually instructed to limit their sodium and fat intake and are generally prescribed ant-hypertensive drugs or anti-hyperlipidemic agents. However, some patients have difficulty fully understanding their physicians' advice, and implementing the dietary suggestions at home. Renal patients being treated on an outpatient basis face the same issues as well. Although they are given nutritional counseling, they have trouble implementing the dietary changes at home. With these challenges in mind, we created an in-hospital restaurant called "Café Marmare" (Figure-2A) to produce meals that meet the criteria and guidelines typical of most lifestyle diseases. In-house dietitians plan the menus, and meals are prepared accordingly by a private contractor. In particular, Café Marmare provides low-sodium and low-fat dishes and 

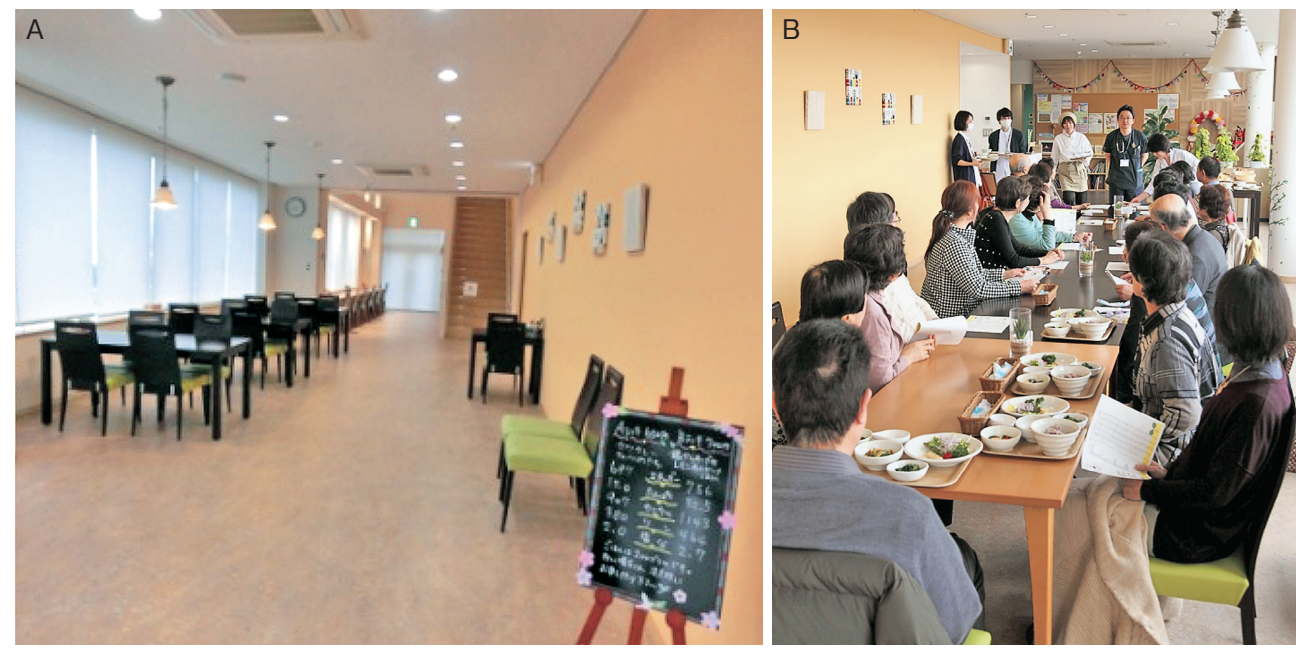

Figure-2

A. Café Marmare, B. Luncheon: hands-on experience to teach patients and their families proper meal planning

incorporates lots of fiber-rich foods in the menu ${ }^{5)}$.

The dietitian on duty each day provides patients and their families with real examples of ideal meal combinations, and suggest healthy cooking methods to meet their individually dietary needs. When necessary dietitians also explains in detail dietary points that were unclear during the patients time with the physicians. These dietitians, as well as physicians, nurses and the medical processor, comprise the restaurant committee and are responsible for determining the operating policies of the restaurant.

Café Marmare provides two lunch sets. Set A is designed to support patients with lifestyle related diseases and contains approximately $2 \mathrm{~g}$ of salt, $200 \mathrm{~g}$ of vegetables and less than $25 \%$ of fat. Set B is designed for individuals in general good health and the dishes within this set are changed weekly. The nutritional information is broken down for both sets so patients can get a concrete idea in terms of taste and volume of what the proper dietary guidelines look like when plated. Patients with renal disease or lifestyle-related diseases visiting Café Marmare state that the low-sodium, fiber rich and the low-fat dishes in restaurant are delicious and motivates them to try making similar dishes at home. Feedback from hospital staff has been positive with reported effects such as change in taste buds and improvements in constipation due to the high fiber contents of the meals.

When visiting the hospital dialysis outpatients can order meals from Café Marmare, and have it delivered to the dialysis room. Approximately half of dialysis outpatients choose this option and we have observed that these patients are less prone to weight gain than patients who make other arrangements for lunch. Luncheons are held in Café Marmare for both dialysis and non-dialysis dependent patients; they are encouraged to bring family members along. At these luncheons patients and their family experience newly devised renal friendly diet plans that they can try to implement at home (Figure-2B).

\section{Kitchen}

The second floor of Café Marmare houses a fully equipped kitchen (Figure-3A). It mainly serves as a staff cafeteria, but is also used for regular cooking classes where hemodialysis and non-dialysis dependent patients and their families can practice preparing food following the guidelines set by the dietitians. Dietitians teach patients how to prepare meals following a theme selected for that day's class such as low-sodium, low-protein, low phosphorous, and constipation prevention (Figure-3B). This hands-on approach allows patients to learn the skills necessary by practicing what is being modeled by dietitians in the kitchen. This makes it more likely that patients will comply with the dietary guidelines at home and makes it easier for them to practice self-management and to successfully make dietary changes for a higher quality of life. So far, these classes are being well received. 

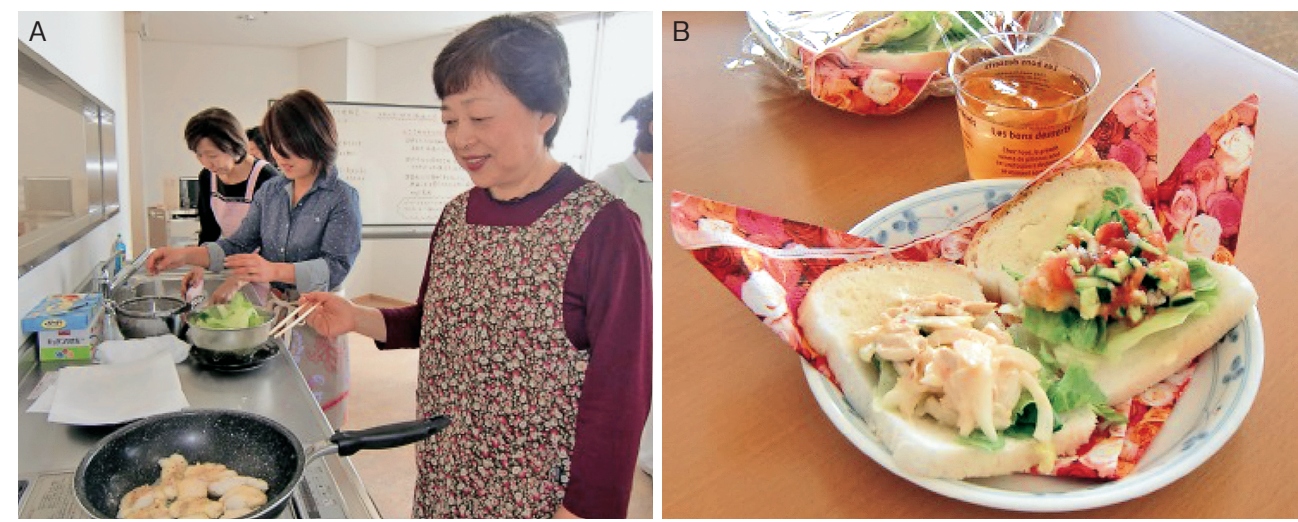

Figure-3

A. Cooking class: preparing low sodium, low fat meals for conservation period patients and their families B. The sandwich contains calorie $595 \mathrm{kcal}$, protein $11.6 \mathrm{~g}$, potassium $350 \mathrm{mg}$ and salt $1.7 \mathrm{~g}$
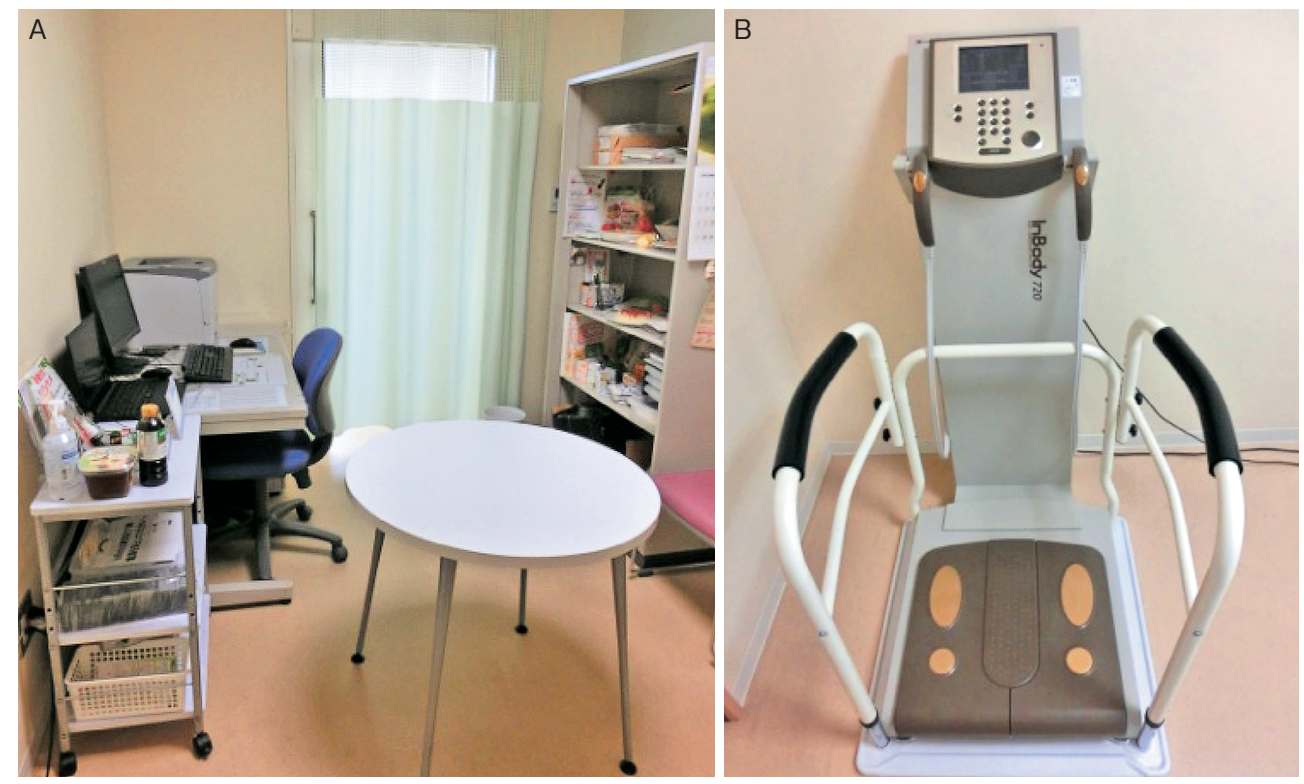

Figure -4

A. Nutrition counseling room, B. Body constituent analyzer in the nutrition counseling room

\section{Nutrition counseling room}

The nutrition counseling room (Figure-4A) is located next to the examination room so that the patients can immediately ask questions and clarify instructions received from physicians and nurses. This type of close cooperation among the hospital staff, makes it possible to provide a strong network of support for patients. A typical flow of nutritional management that a patient can expect is as follows: a physician tests patient's blood and urine samples, prescribes medicine, and gives the patient dietary instructions based on a mode of therapy the physician deems appropriate. Dietitians use this information to calculate the daily sodium and protein intake appropriate for the $\mathrm{CRF}$ patient and if required, they perform a body constituent analysis. Numerical values are provided for items being measured and this allows patients to track their dietary performance and gradually improve their eating habits over time. Keeping track of constituent analysis values not only encourages patients to be accountable for their own health but also helps clinicians track changes in patients' health using indicators such body fat, muscle mass, and water retention volume (Figure-4B). More importantly, constituent analysis tells doctors whether the ingested amount of fat, protein, sodium and water are appropriate for patients and allows 
doctors to make changes until an ideal dietary profile can be created to give the patients the best results.

\section{Other activities}

\section{Nutrition reporting}

As a part of our nutritional outreach program, we now include a section geared towards healthy eating in the "Hotto-Yabuki" which is the hospital's quarterly magazine. In this section, we introduce dietary plans, recipes, and healthy cooking methods and announce related hospital events. Articles in this section offer practical advice for healthy living through dietary reform. One such recent article entitled "Low-Potassium Vegetables" was aimed at readers facing kidney issues. It detailed the fact that CRF patients cannot eliminate potassium properly, therefore they should refrain from consuming raw vegetables since they are abundant in potassium. The article went on to suggest that hydroponic vegetables (grown without soil in water carrying nutrients) are a better option than conventional vegetables since they tend to be low in potassium. The article went on to share the little known fact that Yamagata prefecture had been producing hydroponic vegetables since 2014. This article resulted in an increased understanding of the merits of hydroponic vegetables and motivated $\mathrm{CRF}$ patients to seek these vegetables out.

\section{Supporting athletes}

The need for nutritional support goes beyond the sick and in recent years Yabuki Hospital has undertaken initiatives to support high school athletes such as soccer players and boxers. Before the start of the season for each sport, high school athletes undergo a body constituent analyses, iron level and metabolic tests. Students also fill in questionnaires about their diet and are required to record their food intake for three consecutive days. Students are then paired with a dietitian who interviews the students and obtains additional information. The dietitians analyze this data using nutrient analyzing software and the results are evaluated by a physician. Students and their coaches are given the results and problematic results are discussed in a briefing session with students and their parents.
Our analysis so far suggests that soccer players and boxers tend to lack iron, calcium, and vitamin C and that these athletes tend not to consume enough dietary fiber. We observed also that most female soccer players had iron deficiency so we held cooking classes to help students to use natural foods to alleviate their anemia.

By conducting yearly checkups, there has been a shift in the attitudes of student athletes towards nutrition management, and there is a new awareness among students' family members. Our experience working with these high school athletes has taught us that students' domestic circumstances heavily influences their eating habits and that monitoring students even if once per year motivates them and their parents to make positive health changes not only through their diet but through other lifestyle changes as well.

\section{Supervising menus at restaurants}

For healthy individuals nutrition management is a powerful tool in staving off lifestyle diseases. Especially when dining out extra care is necessary to avoid foods high in sodium and fat. As part of its outreach program Yabuki Hospital is seeking to assist restaurants in the city create healthy menu items. We have successfully concluded a trial at a French restaurant in a local hotel. Our dietitians proposed a full-course meal that was low in sodium $(2.4 \mathrm{~g})$ and total calories $(803 \mathrm{kcal})$. The course was offered for a year and was popular among diners especially those concerned with their blood pressure or weight.

\section{Conclusion}

MNT is an effective and affordable method to supplement medical treatment in CRF patients. Yabuki Hospital has been focusing on nutrition management as a way to improve the quality of life of the renal patients that it serves. In the traditional MNT approach patients are told why and how to change their diet, however Yabuki Hospital takes this a step further and offers renal patients and their family hands-on opportunities to practice the skills they need to successfully implement a diet that works. Since MNT is not only curative but also preventative, the hospital provides nutritional support to high school athletes and has found that 
the feedback and follow-up loop motivates students to be more conscious of their dietary habits. Yabuki Hospital's goal is to expand their activities in the nutrition management field by offering more MNT support to patients and healthy individuals that will increase their quality of life.

\section{Acknowledgement}

I would like to express my gratitude to the dietitians at Yabuki Hospital, Yumiko Seino, Mika Nakajima, Miho Suzuki, Maiko Tsuchiya, Tomoya Sakai and Eriko Suzuki. Their specialist knowledge has helped me prepare this manuscript. I would also like to thank all the hospital staff members for their cooperation and their efforts in creating an ideal nutrition management system.

\section{References}

1) American Diabetes Association: executive summary: standards of medical care in diabetes. Diabetes Care, 2009; 32 Suppl: S6-12.

2) Nakao T, Kanno Y, Nagasawa Y, et al: Diet therapy standard of the chronic dialysis patient. J Jpn Soc Dial Ther, 2014; 47: 287-291. (in Japanese)

3) Masakane I: New Dialysis Therapy from Patient Viewpoint. Tokyo: Shinkoh Igaku Shuppansha, 2011: 31-45. (in Japanese)

4) Yabuki K, Tanida H, Seino Y, et al: Round-table discussion: the role of registered dietitians in nutritional management of dialysis patients. The Japanese Journal of Clinical Nutrition, 2015; 126: 58-63. (in Japanese)

5) Reportage: Division of Health and Nutrition, Yabuki Hospital in Yamagata prefecture. Japanese Journal of Clinical Nutrition, 2016; 128: 86-89. (in Japanese) 